\title{
AVALIAÇÃO DO ENSINO DE RELACIONAMENTO ENFERMEIRA-PACIENTE
}

Maria Lúcia Araújo Sadala*

Maguida Costa Stefanell**

SADALA, M.L.A. ; STEFANELLI, M.C. Avaliação do ensino de relacionamento enfermeira-paciente. Rev.latino.am.enfermagem, Ribeirão Preto, v. 4, $\mathrm{n}^{\circ}$ especial, p. 139-51, abril 1996.

Este trabalho propõe-se a avaliar o ensino de relacionamento enfermeira paciente entre alunas e egressos de Curso de Enfermagem - F. M. Botucatu, UNESP. Os resultados mostram a utilização dos conhecimentos teórico-práticos sobre comunicação relacionamento interpessoal pela população estudada, assim como a avaliação do conteúdo das Disciplinas Relacionamento Enfermeira-Paciente I, II e III do referido curso nos últimos cinco anos.

UNITERMOS: relacionamento enfermeira-paciente, avaliação de ensino

\section{INTRODUÇÃO}

A comunicação é vista na enfermagem como um instrumento básico (HORTA, 1979), mas também como uma competência da enfermeira e uma habilidade a ser desenvolvida (SUNDEEN, 1985; STEFANELLI, 1993) através do aprendizado da teoria da comunicação, técnicas e medidas terapêuticas de enfermagem (STEFANELLI, 1993; SADALA, 1992).

* Professor Doutor do Curso de Enfermagem - F. M. Botucatu. UNESP

** Professor Titular da Escola de Enfermagem da Universidade de São Paulo

Rev. Latino-am.enfermagem - Ribeirão Preto - v. 4 - n. especial - p. 139-151 - abril 1996139 
No Brasil, sabe-se que os cursos de graduação em enfermagem incluem o ensino da teoria de comunicação no conteúdo das disciplinas profissionalizantes, de uma forma genérica, sem sistematização do seu ensino, com raras exceções. No Curso de Enfermagem da F. M. Botucatu - UNESP, criado há 5 anos, a Disciplina Relacionamento Enfermeira-Paciente é ministrada em 3 unidades, de 3 créditos, abrangendo 15 horas de aulas teóricas e 30 horas de aulas práticas, cada uma delas. No presente trabalho, nossa proposta é avaliar o conhecimento teórico-prático sobre comunicação e relacionamento interpessoal enfermeira-paciente assim como o conteúdo ministrado nas disciplinas Relacionamento Enfermeiro-Paciente I, II e III.

\section{METODOLOGIA}

Trata-se de um estudo exploratório descritivo.

Os dados foram obtidos através da aplicação de um questionário (Anexo I), composto por 9 questões abertas, distribuído entre as alunas do $3^{\circ}$ e $4^{\circ}$ anos e as enfermeiras formadas em 1992 e 1993 pelo Curso de Graduação em Enfermagem da F. M. Botucatu-UNESP. 4

\section{RESULTADOS E DISCUSSÃO}

\section{O Aprendizado dos Conceitos}

Participaram do estudo 20 alunas e 12 egressas do referido Curso de Enfermagem, somando 32 respondentes num total de 48 questionários enviados.

As questões n.1 e n.2 do questionário foram elaboradas com o objetivo de avaliar o aprendizado dos conceitos de Comunicação Terapêutica e de Relacionamento Terapêutico. Embora pareçam conceitos semelhantes, a diferenciação nos parece fundamental para a disciplina: a Comunicação Terapêutica é uma habilidade a ser desenvolvida com o aprendizado de comunicação humana e recursos terapêuticos (técnicas e medidas). O Relacionamento Terapêutico consiste num processo com etapas e objetivos planejados e definidos, através de interações estruturadas, cujo sustentáculo é a Comunicação Terapêutica.

As Tabelas 1 e 2 mostram a compreensão dos dois conceitos pelas alunas que participaram do trabalho. 

TABELA 1 - CONCEITO DE COMUNICAÇÃO TERAPÊUTICA SEGUNDO
COMPREENSÃO DAS ALUNAS E EX-ALUNAS. BOTUCATU, 1994

\begin{tabular}{lccccc}
\hline \multicolumn{1}{c}{ CONCEITO } & $\mathbf{3}^{\mathbf{0}}$ Ano & $\mathbf{4}^{\mathbf{0}}$ Ano & $\mathbf{1}^{\text {a }}$ Turma & $\mathbf{2}^{\text {a }}$ Turma & TOTAL \\
\hline $\begin{array}{l}\text { Comunicação para ajudar } \\
\text { o paciente }\end{array}$ & 4 & 7 & 3 & 3 & 17 \\
$\begin{array}{l}\text { Utilização de técnicas } \\
\text { específicas }\end{array}$ & 4 & 4 & 2 & 3 & 13 \\
$\begin{array}{l}\text { Comunicação eficiente } \\
\text { Modo adequado de }\end{array}$ & 5 & 2 & 3 & 4 & 14 \\
$\begin{array}{l}\text { interagir com o paciente } \\
\begin{array}{l}\text { Forma de estimular a } \\
\text { expressão }\end{array}\end{array}$ & 3 & - & - & - & 3 \\
$\begin{array}{l}\text { Habilidade para ajudar o } \\
\text { paciente }\end{array}$ & 1 & - & - & - & 1 \\
Conversar com o paciente & - & - & 1 & - & 1 \\
\hline
\end{tabular}

De acordo com os dados acima as alunas dos quatro grupos conceituam a Comunicação Terapêutica como Comunicação para ajudar o paciente (17 alunos), comunicação eficiente com o paciente (14 alunos) e utilização de técnicas específicas (13 alunos), Algumas alunas (3) consideram-na a forma adequada de interagir com o paciente, forma de estimular a expressão do paciente e habilidade em ajudar o paciente.

Pelos dados da Tabela 2, o relacionamento terapêutico é definido pelas alunas predominantemente como relacionamento de ajuda (17), uma série de interações planejadas (13) e por algumas como a utilização da comunicação terapêutica (4), contrato para ajudar o paciente (2), e relacionamento consciente (2).

Observando-se os dados acima, as alunas diferenciam explicitamente a comunicação terapêutica do relacionamento terapêutico, a primeira como uma forma de comunicação de ajuda que utiliza técnicas específicas, possibilitando a comunicação eficiente. O relacionamento é delimitado como um relacionamento com o objetivo de ajudar o paciente, com uma forma estruturada através de interações planejadas, utilizando-se os conhecimentos da comunicação terapêutica. 
TABELA 2 - CONCEITO DE RELACIONAMENTO TERAPÊUTICO SEGUNDO COMPREENSÃO DAS ALUNAS E EX-ALUNAS. BOTUCATU, 1994

\begin{tabular}{lcccc}
\hline \multicolumn{1}{c}{ CONCEITO } & 4 $^{\text {0 Ano }}$ & $\mathbf{1}^{\text {a }}$ Turma & $\mathbf{2}^{\text {a }}$ Turma & TOTAL \\
\hline Relacionamento de ajuda & 6 & 6 & 5 & 17 \\
Série de interações planejadas & 4 & 4 & 5 & 13 \\
Utilização da comunicação terapêutica & 2 & - & 2 & 4 \\
Contrato para ajudar o paciente & 1 & - & 1 & 2 \\
$\begin{array}{l}\text { Relacionamento consciente com objetivo } \\
\text { definido }\end{array}$ & 1 & 1 & - & 2 \\
Troca de experiência & 1 & - & - & 1 \\
Relacionamento de confiança & 1 & - & - & 1 \\
\hline
\end{tabular}

\section{Utilização das Técnicas}

Todos os respondentes dos 4 grupos informaram que têm utilizado as técnicas de comunicação terapêutica e as medidas terapêuticas de enfermagem ao cuidar dos pacientes, com exceção de uma aluna do $3^{\circ}$ ano que diz usá-la às vezes. As técnicas e medidas utilizadas estão descritas n.1 Tabela 3.

\begin{tabular}{|c|c|c|c|c|c|}
\hline $\begin{array}{c}\text { TÉCNICAS E MEDIDAS } \\
\text { UTILIZADAS }\end{array}$ & $3^{\circ}$ Ano & $4^{\circ}$ Ano & $1^{\mathrm{a}}$ Turma & $2^{\mathrm{a}}$ Turma & TOTAL \\
\hline Saber ouvir & 7 & 7 & 5 & 4 & 23 \\
\hline Verbalizar interesse & 8 & 6 & 3 & 3 & 20 \\
\hline Verbalizar aceitação & 6 & 4 & 3 & 3 & 16 \\
\hline Utilização de perguntas & 9 & 5 & 3 & 4 & 21 \\
\hline Utilização de frases descritivas & 7 & 5 & - & - & 12 \\
\hline Uso do silêncio terapêutico & 7 & - & 4 & 3 & 14 \\
\hline Dizer não & 5 & - & - & - & 5 \\
\hline Devolver a pergunta & 2 & 1 & 2 & 1 & 6 \\
\hline Repetir o que foi dito & - & 1 & - & - & 1 \\
\hline Verbalizar dúvidas & - & 1 & - & - & 1 \\
\hline Clarificar & 3 & - & - & 1 & 4 \\
\hline Validar & 1 & - & 2 & 1 & 4 \\
\hline Colocar em foco a idéia principal & - & - & 2 & - & 2 \\
\hline Oferecimento de apoio & 9 & 4 & 3 & 4 & 20 \\
\hline Estabelecimento de limites & 7 & 3 & 2 & 3 & 15 \\
\hline Estimular a expressão & - & 2 & 2 & 4 & 8 \\
\hline
\end{tabular}


As técnicas mais utilizadas pelas alunas são as técnicas que ajudam a expressão do paciente: saber ouvir, verbalizar interesse e aceitação, utilização de perguntas, utilização de frases descritivas, uso do silêncio terapêutico e dizer não. Clarificar (4) e validar (4) foram citados poucas vezes. Estes resultados corroboram os dados obtidos por STEFANELLI (1993) e SADALA (1992) em pesquisa sobre a utilização da comunicação terapêutica por estudantes de enfermagem. As do grupo expressão são as mais usadas no início de um relacionamento: histórico de enfermagem, dados para o diagnóstico, o que o paciente sente e pensa sobre a experiência da internação e tratamento.

O oferecimento de apoio (20) e o estabelecimento de limites (15) são as medidas terapêuticas mais citadas. Estimular a expressão do paciente aparece 8 vezes, dado que corrobora a predominância das respostas sobre as técnicas mais utilizadas.

As alunas citam as situações nas quais utilizam a comunicação terapêutica na seguinte ordem decrescente: na assistência ao paciente (14), quando o paciente se mostra ansioso (5), sempre que necessário (4), orientações ao paciente (2), entrevistas com o paciente (3), aluna ansiosa (2) e entrevistas com o paciente (2). No geral, trata-se de situações de assistência de enfermagem, o que não deixa de ser um indicador do desenvolvimento da habilidade no seu uso, ou seja, em qualquer ação de enfermagem e não desvinculada do todo. No entanto, quando as alunas delimitam as situações específicas - como as citadas, parece-nos ser necessário discutirmos melhor em classe a questão "sempre que necessário": não se preconiza o uso da comunicação terapêutica como ação isolada.

\section{Dificuldades na prática dos conhecimentos teóricos}

As dificuldades citadas pelos quatro grupos de alunas (Tabela 4), foram mais citadas na seguinte ordem: falta de domínio do processo de comunicação (8), onde predominam respostas das alunas do $3^{\circ}$ ano (4); estabelecer os limites para o paciente (8), onde predominam respostas de alunas do $3^{\circ}$ e $4^{\circ}$ ano $(2$ e 3 respectivamente); ansiedade da aluna (6), onde predominam respostas das alunas do $3^{\circ}$ ano (3); o oferecimento de apoio (6), onde predominam respostas das alunas de $3^{\circ}$ ano (4). Observa-se pelos dados apresentados que as dificuldades são mais lembradas pelas alunas de $3^{\circ}$ ano, o que pode ser interpretado como resultado de uma experiência mais limitada em aplicar os conhecimentos da comunicação terapêutica, mesmo porque a essas alunas não foi ministrada a. disciplina Relacionamento Enfermeiro-Paciente III, que trata especificamente da sistematização do processo de relacionamento terapêutico. 
TABELA 4 - DIFICULDADES NA UTILIZAÇÃO DA COMUNICAÇÃO TERAPÊUTICA APONTADAS PELAS ALUNAS E EX-ALUNAS. BOTUCATU,1994

\begin{tabular}{lccccc}
\hline \multicolumn{1}{c}{ DIFICULDADES } & $\mathbf{3}^{\mathbf{0}}$ Ano & $\mathbf{4}^{\mathbf{0}}$ Ano & $\mathbf{1}^{\mathbf{a}}$ Turma & $\mathbf{2}^{\mathbf{a}}$ Turma & TOTAL \\
\hline Estimular a expressão & - & 1 & - & - & 1 \\
Oferecimento de apoio & 4 & 1 & 1 & - & 6 \\
Estabelecimento de limites & 2 & 3 & 1 & 2 & 8 \\
Ansiedade da aluna & 3 & 1 & 2 & - & 6 \\
Falta de domínio do processo & 4 & 2 & 1 & 1 & 8 \\
de comunicação & & & & & \\
Dinâmica de assistência de & - & 2 & 2 & - & 4 \\
enfermagem & - & - & - & 1 & 1 \\
Desconhecimento do paciente & - & - & - & 1 & 1 \\
Resistência dos pacientes & - & - & - & 1 & 1 \\
Falta de empatia & 2 & - & - & 2 & - \\
Não tem dificuldade & & & & & \\
\hline
\end{tabular}

Algumas alunas ainda citam como dificuldade a dinâmica da assistência de enfermagem, onde estão incluídos a troca freqüente de pacientes e a falta de privacidade para interagir com os mesmos, o que dificulta desenvolver 0 relacionamento com o paciente, Precisamos reforçar que a utilização da comunicação terapêutica deve culminar até em estilo de vida, e, portanto, não se deve escolher momentos para usá-la e sim fazer de cada situação um momento terapêutico.

Três alunas citam variáveis do paciente que dificultam o processo de comunicação: desconhecimento do paciente, resistência do paciente em interagir e falta de envolvimento do paciente, Duas alunas dizem não ter dificuldade em utilizar a comunicação terapêutica.

\section{Desenvolvimento do relacionamento terapêutico}

A questão que se refere ao desenvolvimento do Relacionamento Terapêutico pelas alunas foi respondida pelos grupos do $4^{\circ}$ ano e ex-alunas da $1^{a}$ e $2^{\mathrm{a}}$ turma, pois. o tema é abordado a partir do $3^{\circ}$ ano, na disciplina Relacionamento III.

Todas as alunas informam que desenvolveram o processo de relacionamento interpessoal com pacientes, durante a execução de atividades em diversas especialidades descritas na tabela a seguir. 
TABELA 5 - LOCAIS NOS QUAIS ALUNAS E EX-ALUNAS DESENVOLVERAM O RELACIONAMENTO INTERPESSOAL COM O PACIENTE. BOTUCATU, 1994

\begin{tabular}{|c|c|c|c|c|}
\hline LOCAIS & $4^{\circ}$ Ano & $1^{\text {a }}$ Turma & $2^{\mathrm{a}}$ Turma & TOTAL \\
\hline Isolamento & 6 & - & 5 & 11 \\
\hline Centro-cirúrgico & 6 & - & - & 6 \\
\hline Ginecologia & 4 & 1 & - & 5 \\
\hline Obstetrícia & 3 & 1 & - & 4 \\
\hline Neonatologia & 2 & - & - & 2 \\
\hline Pediatria & 3 & - & 3 & 6 \\
\hline Médico Cirúrgica & 3 & 2 & 2 & 7 \\
\hline Psiquiatria & 1 & 2 & 5 & 8 \\
\hline Administração & - & 2 & - & 2 \\
\hline Enfermaria onde trabalha & - & 2 & - & 2 \\
\hline $\begin{array}{l}\text { Em todas as disciplinas, sem completar todas } \\
\text { as frases }\end{array}$ & - & 1 & 1 & 2 \\
\hline
\end{tabular}

As situações mais citadas pelas alunas correspondem às atividades de estágio no isolamento (11), na psiquiatria (8), em enfermaria médico-cirúrgica (7), em pediatria (6) e centro-cirúrgico (6). Estas atividades são desenvolvidas concomitantemente às disciplinas Relacionamento II e Relacionamento III, observando-se que na enfermaria médico-cirúrgica o relacionamento foi desenvolvido de forma incompleta. Pode-se dizer, além disso, que nas atividades citadas as alunas tiveram a oportunidade de estarem mais tempo junto ao paciente (especialmente no isolamento e na psiquiatria). De acordo com os dados, pode-se considerar que as alunas desenvolveram o relacionamento interpessoal com os pacientes quando estavam sob a supervisão da docente das disciplinas Relacionamento I, II e III, e, no caso da psiquiatria, sob a supervisão das docentes da Psiquiatria.

\section{DIFICULDADES AO DESENVOLVER O RELACIONAMENTO TERAPÊUTICO}

As dificuldades citadas pelas alunas ao desenvolverem o relacionamento terapêutica com os pacientes foram classificadas nas categorias seguintes: ansiedade da aluna, percebida por 10 respondentes, 5 das quais da $2^{\mathrm{a}}$ turma; sistematização $\mathrm{d}($ ) relacionamento, que abrangem dificuldades relativas ao planejamento das interações, início e término do relacionamento que foram lembrados por 5 alunas, 3 do $4^{\circ}$ ano; falta de envolvimento do paciente, citada 5 vezes sendo 3 de egressas, abrangendo insegurança e não aceitação do relacionamento por parte dos pacientes. 
A dinâmica da assistência de enfermagem no H.C. com a troca freqüente de pacientes e o ambiente hospitalar foram citadas por 3 alunas como variáveis que dificultaram o desenvolvimento do relacionamento terapêutico com os pacientes. A falta de experiência, o medo de ser não terapêutica e a dificuldade de ser supervisionada ao desenvolver o processo foram citadas por 2 alunas.

É de fundamental importância analisar cada uma das situações acima descritas para repensarmos nossa disciplina.

\section{Sentimentos das alunas influindo no processo de comunicação com o paciente}

Nesta questão procuramos investigar a percepção que as alunas têm de si mesmas ao interagirem com os pacientes, assim como a influência que esta variável representa na utilização da comunicação terapêutica. A ansiedade foi citada por 13 alunas, ansiedade por desconhecer o paciente, pelas diferenças sócio-culturais, ao colocar limites, ao finalizar o relacionamento; 7 alunas apontam a insegurança ao iniciar o relacionamento, por não saberem como continuá-lo e ao colocarem limites; 6 alunas citam o me(/o de rejeição, de não corresponder às expectativas do paciente, de paciente de isolamento, de ser preconceituosa, de se envolver excessivamente. Percebe-se que os três sentimentos citados são próximos entre si, se não evolutivos, um contém os outros. As alunas porém os identificaram desta forma e é importante que se note a sua capacidade de diferenciar tais percepções: os objetos dos sentimentos e as reações que provocam são distintos.

Outros sentimentos são citados: envolvimento excessivo com paciente, (5 vezes); o envolvimento (3 vezes) e a compaixão (2 vezes) lembrados como sentimentos positivos que possibilitaram o aparecimento de empatia com 0 paciente. Tivemos ainda a frustração, a impaciência e a angústia citados uma 1 vez.

\section{Avaliação do conteúdo da disciplina}

As respostas positivas a respeito do conteúdo das disciplinas Relacionamento I, II e III são apresentadas na Tabela 6. 
TABELA 6 - AVALIAÇÃO DO CONTEÚDO DAS DISCIPLINAS RELACIONAMENTO ENFERMEIRA-PACIENTE I, II E III PELA POPULAÇÃO DO ESTUDO. BOTUCATU, 1994

\begin{tabular}{lccccc}
\hline \multicolumn{1}{c}{ AVALIAÇÃo } & $\mathbf{3}^{\mathbf{0}}$ Ano & $\mathbf{4}^{\mathbf{0}}$ Ano & $\mathbf{1}^{\text {a }}$ Turma & $\mathbf{2}^{\mathbf{a}}$ Turma & TOTAL \\
\hline Fundamental para a prática de enfermagem & 11 & 1 & 3 & 1 & 16 \\
Bom, muito bom, excelente & 2 & 6 & 1 & 5 & 14 \\
Muito útil & 1 & - & - & - & 1 \\
Adequado para a prática de enfermagem & - & 2 & 2 & 2 & 6 \\
$\begin{array}{l}\text { Ensino de técnicas para relacionar-se com o } \\
\text { paciente }\end{array}$ & 2 & 3 & 1 & - & 6 \\
$\begin{array}{l}\text { Reflexão sobre comunicação com o paciente } \\
\text { Dá segurança na comunicação com o }\end{array}$ & 1 & - & - & 1 & 2 \\
paciente & 1 & - & - & - & 1 \\
$\begin{array}{l}\text { Diferencia a assistência de enfermagem } \\
\text { Ensina a ser gente }\end{array}$ & 1 & 1 & - & - & 2 \\
$\begin{array}{l}\text { Algumas vezes repetitivo } \\
\text { Prática não satisfatória }\end{array}$ & - & 1 & - & - & 1 \\
Ensina o relacionar-se como aluno, ser & - & - & 1 & - & 1 \\
humano e como profissional & - & - & 1 & - & 1 \\
Suficiente & & - & 1 & - & 1 \\
\hline
\end{tabular}

Consideramos importante ressaltar que algumas respostas, embora aparecendo um número pequeno de vezes, nos desafiam a investigar mais atentamente alguns pontos, como: "o conteúdo é algumas vezes repetitivo", chamando a nossa atenção para o objetivo das disciplinas ministradas em 3 unidades: cada uma deverá acrescentar à anterior, mas deve evitar ser repetitiva. "A prática não satisfatória", citada uma vez, também nos chama a atenção para a necessidade de adequação dos conteúdos à aplicação no dia a dia da enfermeira. As sugestões da população são apresentadas na Tabela 7.

TABELA 7 - SUGESTÕES PARA MELHORAR O ENSINO DAS DISCIPLINAS. BOTUCATU, 1994

\begin{tabular}{lccccc}
\hline \multicolumn{1}{c}{ SUGESTÕES } & $\mathbf{3}^{\mathbf{0}}$ Ano & $\mathbf{4}^{\mathbf{0}}$ Ano & $\mathbf{1}^{\text {a }}$ Turma & $\mathbf{2}^{\mathbf{a}}$ Turma & TOTAL \\
\hline Mais atividades práticas & 5 & 2 & 4 & 4 & 15 \\
Continuar como está & 3 & 3 & - & 2 & 8 \\
Trabalho em grupo & 1 & 2 & - & - & 3 \\
Maior tempo para a disciplina & 1 & - & - & - & 1 \\
A disciplina em todas as escolas de & 1 & - & - & - & 1 \\
enfermagem do Brasil & - & 2 & - & - & 2 \\
Condensar a disciplina em dois semestres & - & - & 1 & - & 1 \\
Mais interação com outras disciplinas & - & - & 1 & - & 1 \\
Manter os alunos mais tempo com um & - & - & - & 1 & 1 \\
paciente & - & - & - & 1 & 1 \\
Maior prática de relacionamento & & & & 1 \\
Maior referência bibliográfica & & & & \\
\hline
\end{tabular}


Aqui destacam-se predominantemente as duas primeiras sugestões: mais atividades práticas (15 vezes) e continuar como está (8 vezes), embora a primeira seja quase o dobro.

As atividades práticas sugeridas incluem: mais trabalhos práticos, mais aulas práticas, pesquisas, mais análises de interações, estágio específico para a disciplina e a docente da disciplina participar dos estágios curriculares.

As disciplinas de Relacionamento Enfermeiro-Paciente são ministradas concomitantemente aos estágios curriculares de Fundamentos de Enfermagem, Enfermagem Médico-Cirúrgica, Enfermagem em Doenças Transmissíveis e em Centro Cirúrgico. O trabalho prático de relacionamento que os alunos desenvolvem são realizados nas disciplinas correspondentes, e supervisionados por nós através do registro e análise das interações. Somente em situações especiais acompanhamos os alunos nos estágios curriculares. Devemos lutar pela implementação de atividades junto às enfermeiras que atuem nos campos de estágio e, também, aos docentes da Escola.

\section{CONSIDERAÇÕES FINAIS E CONCLUSÕES}

Podemos considerar, após a análise dos dados deste estudo, que as alunas e ex-alunas das disciplinas Relacionamento Enfermeiro-Paciente I, II e III ministradas nos últimos 5 anos:

- descrevem os conceitos de comunicação Terapêutica e Relacionamento Terapêutico de forma prática e definem especificamente cada um deles, embora de forma fragmentada às vezes;

- afirmam utilizar técnicas de comunicação terapêutica, predominantemente as técnicas que ajudam a expressão do paciente e as medidas terapêuticas de enfermagem de modo geral, ao prestarem assistência ao paciente;

- percebem as suas dificuldades ao utilizarem os conhecimentos teóricos sobre comunicação, sendo as mais citadas: estabelecer limites para o paciente, falta de domínio dos conhecimentos sobre o processo de comunicação, oferecimento de apoio e ansiedade da aluna;

- a partir do $4^{\circ}$ ano, procuram desenvolver relacionamento terapêutico em todas as enfermarias, conseguindo atingir o objetivo predominantemente nas atividades desenvolvidas com o paciente em isolamento, internado em unidade psiquiátrica; em enfermagem de centro-cirúrgico e médico-cirúrgica;

- descrevem os sentimentos que interferem na comunicação com o paciente, predominando a ansiedade, a insegurança e o medo;

- o conteúdo das disciplinas é avaliado positivamente e considerado importante para a prática da enfermagem; 
- sugerem a implementação das atividades práticas nas enfermarias, no sentido de mais oportunidades para desenvolverem a comunicação com os pacientes.

Estes dados representam uma análise importante da disciplina, no sentido de proporcionar a visão das possibilidades e dificuldades da utilização da teoria na prática, desta forma oferecendo elementos para a reelaboração do conteúdo, procedimentos pedagógicos e atividades propostas pelas disciplinas Relacionamento Enfermeiro-Paciente I, II e III, introduzidas no currículo do Curso de Enfermagem da F.M, de Botucatu por Máguida Costa Stefanelli e ministradas por Maria Lúcia Araújo Sadala.

\title{
EVALUATION OF TOE TEACHING OF COMMUNICATION SKILLS IN NURSING UNDERGRADUATE AND GRADUATE COURSE
}

This study aims at evaluating the learning of communication skills by undergraduate and graduate students from the School of Nursing at Botucatu, São Paulo, in the last five years,

UNITERMS: nursing education, learning of communication skills, evaluation of education

\section{EVALUACIÓN DE LA ENSEÑANZA DE RERACIONAMIENTO ENFERMERO-PACIENTE}

\begin{abstract}
El presente trabajo se propone evaluar la enseñanza del reracionamiento enfermero-paciente entre los alumnos y graduados del Curso de Enfermería de la F. M. Botucatu - UNESP, Los resultados muestran la utilización de los conocimientos teórico-prácticos sobre la comunicación y relacionamiento interpersonal por la población estudiada, así como la evaluación del contenido de las disciplinas Relacionamiento Enfermero-Paciente I, II y III del referido curso por un período de cinco años,
\end{abstract}

UNITERMOS: relacionamiento enfermero-paciente, evaluación de enseñanza 


\section{REFERÊNCIAS BIBLIOGRÁFICAS}

01. HORTA, W.A. O processo de enfermagem. São Paulo: EPU, 1979.

02. SADALA, M.L.A. Desenvolvendo a habilidade em comunicação. São Paulo, 1992. /Trabalho apresentado ao Congresso Internacional de Pesquisa em Enfermagem, Escola de Enfermagem da Universidade de São Paulo, São Paulo/.

03. STEFANELLI, M.C. Comunicação com o paciente: teoria e ensino. 2ed. São Paulo: Robe, 1993.

04. SUNDEEN, S.J. et al. Nursing client interaction: implementing the nursing process. 3 ed. St. Louis: C.V. Mosby, 1985. 


\section{ANEXO I}

\section{QUESTIONÁRIO DESTINADO AOS ALUNOS DO CURSO DE GRADUAÇÃO EM ENFERMAGEM SOBRE A DISCIPLINA RELACIONAMENTO ENFERMEIRO-PACIENTE}

1. Como você conceitua, com suas palavras, o que é a comunicação terapêutica?

2. Como você conceitua, com suas palavras, o que é relacionamento terapêutico?

3. Você tem utilizado as técnicas de comunicação terapêutica e as medidas terapêuticas da enfermagem ao prestar assistência aos pacientes? Cite as mais usadas e em que situação as utilizou?

4. Descreva as dificuldades que você encontra ao utilizar ou tentar utilizar as técnicas de comunicação terapêutica e as medidas terapêuticas (oferecimento de apoio, estabelecimento de limites e ajudar na expressão dos pensamentos e sentimentos).

5. Você conseguiu desenvolver Relacionamento Terapêutico com algum (ou alguns) pacientes que esteve sob seus cuidados? Em que disciplinas?

6. Quais as dificuldades que você encontrou ao desenvolver, ou tentar desenvolver, Relacionamento Terapêutico com o paciente?

7. Descreva os sentimentos experimentados por você que possam ter interferido na comunicação com o paciente.

8. Como você considera o conteúdo ministrado na disciplina Relacionamento Enfermeiro-Paciente, para a Prática de Enfermagem?

9. Faça as sugestões que julgar pertinentes para o aprimoramento da disciplina Relacionamento Enfermeiro-Paciente. 